\title{
Low-Power Adiabatic Computing with Improved Quasistatic Energy Recovery Logic
}

\author{
Shipra Upadhyay, R. K. Nagaria, and R. A. Mishra \\ Department of Electronics and Communication Engineering, Motilal Nehru National Institute of Technology, Allahabad 211004, India \\ Correspondence should be addressed to Shipra Upadhyay; shipraupadhyay2@gmail.com
}

Received 30 May 2013; Accepted 27 August 2013

Academic Editor: Jose Silva-Martinez

Copyright ( 2013 Shipra Upadhyay et al. This is an open access article distributed under the Creative Commons Attribution License, which permits unrestricted use, distribution, and reproduction in any medium, provided the original work is properly cited.

\begin{abstract}
Efficiency of adiabatic logic circuits is determined by the adiabatic and non-adiabatic losses incurred by them during the charging and recovery operations. The lesser will be these losses circuit will be more energy efficient. In this paper, a new approach is presented for minimizing power consumption in quasistatic energy recovery logic (QSERL) circuit which involves optimization by removing the nonadiabatic losses completely by replacing the diodes with MOSFETs whose gates are controlled by power clocks. Proposed circuit inherits the advantages of quasistatic ERL (QSERL) family but is with improved power efficiency and driving ability. In order to demonstrate workability of the newly developed circuit, a $4 \times 4$ bit array multiplier circuit has been designed. A mathematical expression to calculate energy dissipation in proposed inverter is developed. Performance of the proposed logic (improved quasistatic energy recovery logic (IQSERL)) is analyzed and compared with CMOS and reported QSERL in their representative inverters and multipliers in VIRTUOSO SPECTRE simulator of Cadence in $0.18 \mu \mathrm{m}$ UMC technology. In our proposed (IQSERL) inverter the power efficiency has been improved to almost $20 \%$ up to $50 \mathrm{MHz}$ and $300 \mathrm{fF}$ external load capacitance in comparison to CMOS and QSERL circuits.
\end{abstract}

\section{Introduction}

With increased scaling in CMOS technology, modern designs are capable of performing very high speed computations as the complexity, and the number of devices on a given IC is no longer an issue. Much of the research efforts in the recent decades have been dedicated to improving the speed of digital systems. Thus, high speed computation has become an expected norm for average users. Higher switching activities lead to higher power consumption. Many methodologies have been proposed so far [1] which intended to reduce power consumption, among them adiabatic logic technique [2] is promising alternative. Concept of adiabatic logic circuits is generated from the adiabatic process which is a thermodynamically reversible process that is operated slowly, so that total energy dissipation tends towards zero. Energy dissipated in a circuit depends on how fast the circuit switches or charges and discharges which means that it depends on the approach taken to design the circuit. When the rate of charging will be lower, less amount of energy is drawn from the source. Adiabatic circuits also have another mechanism for energy saving
[3] that is based on recovering the energy stored in nodal capacitances. The quality factor $Q$ of any adiabatic process is also known as degree of adiabaticity or adiabatic gain, and it is the ratio between the total energy delivered and the energy that gets dissipated in the whole process.

There are three types of losses in adiabatic circuits, nonadiabatic losses, adiabatic losses, and leakage losses. The last two losses are associated with fully adiabatic circuits, whereas nonadiabatic losses are related to quasi/semiadiabatic circuits. Nonadiabatic losses are proportional to the voltage drop across the terminals of a resistive switch, when it is on $[4,5]$. Leakage losses are proportional to the clock period and are negligible in comparison to the other two. The third one is adiabatic loss which depends on current or voltage drawn from the source, load capacitance, charging path resistance, and transition time [6, 7]. It should be noted that nonadiabatic losses can be eliminated completely by using reversible logic, but adiabatic and leakage losses cannot be avoided. There are several remedies to reduce adiabatic losses. First one is by extending the charging time $T$. The second way is constant current charging [8] for capacitance through 
a resistance in a given time. Third method is by lowering the charging path resistance, which was not the case in CMOS.

Another important mechanism for energy saving in adiabatic circuits is to recover the adiabatic losses. The energy which was stored in load capacitance during charging can be recovered again with a dissipation that depends inversely on charging time. This is possible if the current direction is reversed, and load capacitance is discharged through the same path. The difference between the nodal energy and dissipated part is recovered from the current source and can be used in next charging. For this purpose, the DC power source of CMOS is replaced by ramp, sinusoidal, trapezoidal, and triangular and so forth the power clock signals in adiabatic circuits $[9,10]$. Initially most adiabatic circuits were based on multiphase power clocks. But they were not suitable for high speed design, because of their complex controlling mechanism. Comparatively single phase adiabatic circuits are suitable for operating at high speeds with high energy efficiency, because they have simple clock controlling mechanism. Considering these facts, we propose IQSERL circuit which inherits all the advantages of recent reported circuits [9] with additional improvement in energy efficiency and speed.

The paper is organized into five sections as follows. Section 1 deals with the introduction part. Section 2 describes the limitations of QSERL circuits. In Section 3, we have discussed the proposed energy recovery logic (IQSERL) inverter circuit. The result and performance analysis of IQSERL based inverter and $4 \times 4$ bit array multiplier circuit are carried out in Section 4. Section 5 summarizes the conclusion.

\section{Limitations in QSERL Circuits}

Many adiabatic circuits proposed over years based on multiphase clocking scheme suffer from several drawbacks like complex circuit structure, poor driving ability, inrobustness, and large delay. The trapezoidal power clock based efficient charge recovery logic (ECRL) [11], clocked adiabatic logic circuits (CAL) [12], and positive feedback adiabatic logic (PFAL) circuits [13] suffer from floating output nodes, problem of current leakage, and charge sharing. This limits their use at high frequency, and thus operating speed is slow.

Glitch free cascadable adiabatic logic (GFCAL) [10] circuit is based on triangular power clock which have lowest power dissipation among all, but their main drawback is very large delay and hence very slow operating speed. Sinusoidal power clock based adiabatic circuits are QSERL, complementary energy path adiabatic logic (CEPAL) [14], and twophase adiabatic static CMOS logic (2PASCL) circuits [15]. The 2PASCL has comparatively simple circuit structure and lower switching activity, but there exists a small amplitude ripple on both high and low logic voltage levels which was caused by the on/off resistances of switching transistors and load capacitances. Thus output amplitude is degraded.

QSERL circuit has hold phase due to this dynamic switching, and thus energy dissipation is reduced, but output is floating which is not desirable. CEPAL circuit attempts to improve and remove the drawback of QSERL circuit by removing the hold phase. Its throughput is also better than QSERL and is twice. But it is comparatively less power efficient than QSERL. The significant delay derived from QSERL based combinational logic is very important thing to be discussed and remedied. Referring to Figure 1, an eight-stage inverter chain has been simulated with $50 \mathrm{MHz}$ input rate and $100 \mathrm{MHz}$ power clock rate, and it has significant amount of delay at each stage which causes completely incorrect output logics at the seventh and eighth-stage. It makes QSERL slightly disadvantageous.

\section{Improved Quasistatic Energy Recovery Logic (IQSERL) Circuits}

3.1. Circuit Description. The schematic of IQSERL circuit is shown in Figure 2. It is composed of a P network, an N network, two complementary split-level sinusoidal power clocks $\left(V_{\Phi}\right.$ and $\left.V_{\bar{\phi}}\right)$, charging $\mathrm{pMOS}$ transistor (P1), and discharging nMOS transistor (N1) whose gates are controlled by the power clocks. Both the power clocks are 180 degree out of phase. The voltage level of the clock $V_{\Phi}$ exceeds that of inverted clock $V_{\bar{\phi}}$ by a factor of $V_{\mathrm{dd}} / 2$, this will minimize the voltage difference between the electrodes and consequently power dissipation. Split-level sinusoidal clock charges/discharges the load capacitances comparatively slowler than the other adiabatic power clocks.

This is desirable because efficiency of adiabatic logic circuits depends upon how slowly the load capacitance is charged/discharged. The peak-to-peak voltage of these power clocks $V_{\Phi}$ and $V_{\bar{\phi}}$ is $0.9 \mathrm{~V}$ :

$$
\begin{gathered}
V_{\Phi}=\frac{V_{\mathrm{dd}}}{4} \sin (\omega t+\theta)+\frac{3}{4} V_{\mathrm{dd}} \\
V_{\bar{\phi}}=-\frac{V_{\mathrm{dd}}}{4} \sin (\omega t+\theta)+\frac{1}{4} V_{\mathrm{dd}} .
\end{gathered}
$$

The pMOS (P1) in the pull up network and nMOS (N1) in the pull down network are used to replace the diode used in QSERL circuit for the charging and discharging, respectively. Power clock $\left(V_{\Phi}\right)$ controls the switching on and off of transistors (N1), and $V_{\bar{\phi}}$ controls the switching on and off of transistors (P1). These power clocks can be generated and realized practically by a simple LC resonant oscillator as shown in [15]. The nonadiabatic source of power dissipation in reported QSERL circuits in charging and discharging path occurs at the (MOSFET) diodes due to the threshold voltage drop, whereas in our proposed circuit, power dissipation is due to the adiabatic losses of on resistance of channels of MOSFET transistors.

As we discussed in previous sections that in irreversible circuits the nonadiabatic losses cannot be eliminated, whereas adiabatic losses can be reduced by several remedies, thus the drain to source voltage drop of charging/discharging transistors and hence power dissipation may be reduced by slowing down the charging/discharging time. However, because of irreversibility [16], it cannot be removed completely. 


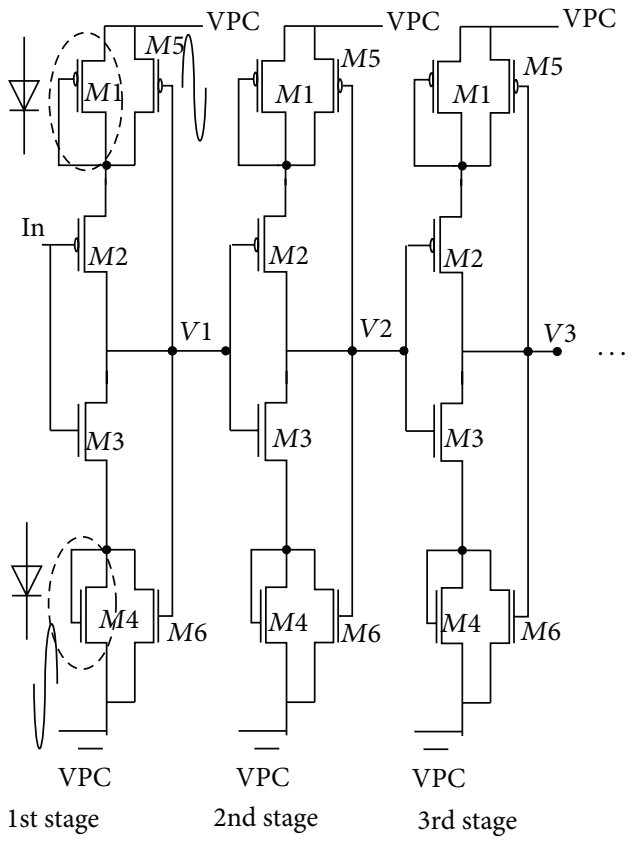

(a)

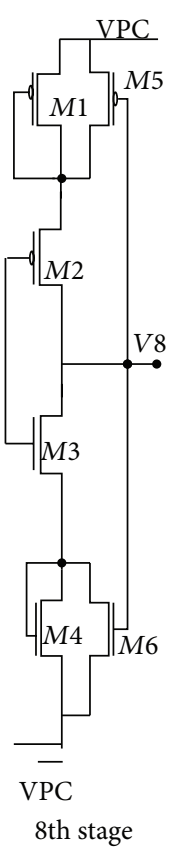

8th stage

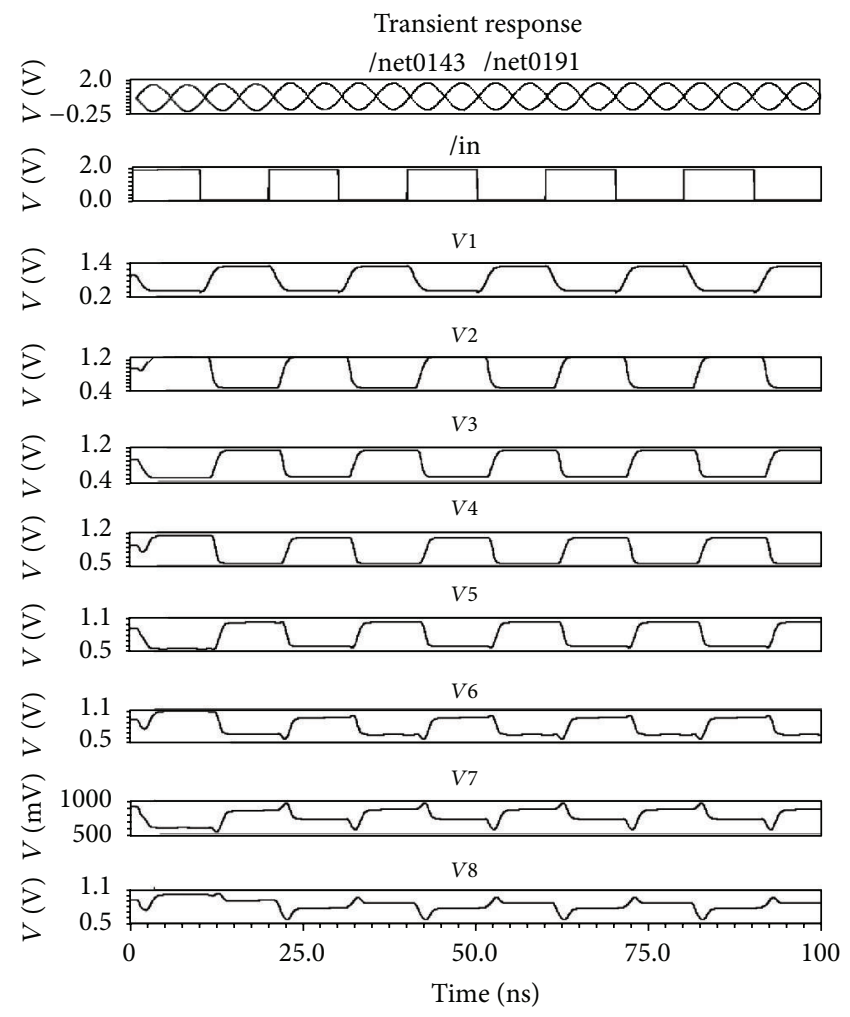

(b)

FIGURE 1: (a) QSERL eight-stage inverter chain, (b) transient response.

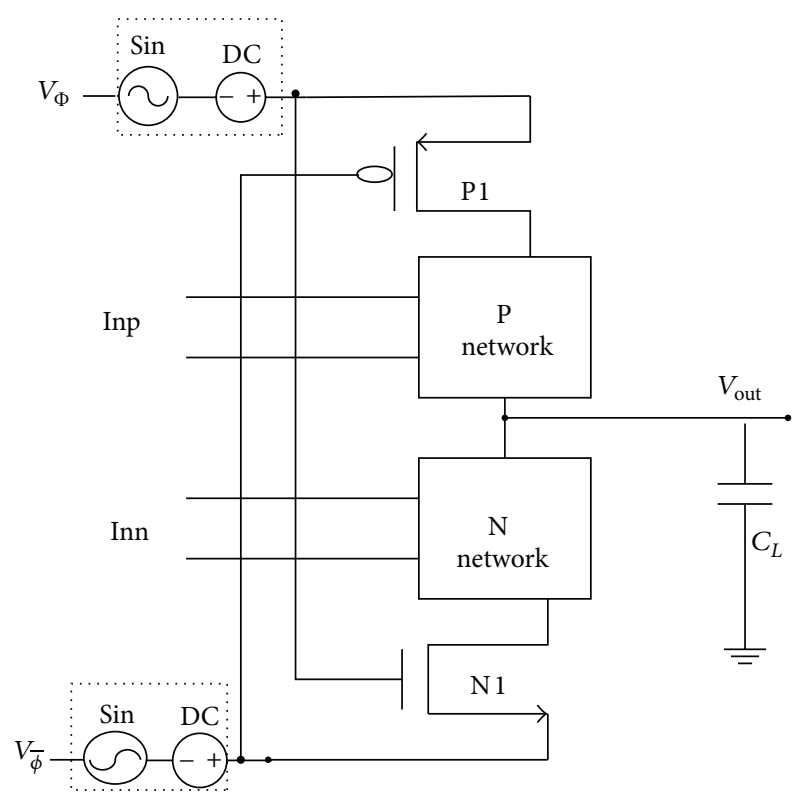

FIgURe 2: Proposed IQSERL circuit.

3.2. Circuit Operation. Based on the supply clock structure, operation of IQSERL circuit is divided into two phases, evaluation and hold. In evaluation phase $V_{\Phi}$ goes up, while $V_{\bar{\phi}}$ goes down; however, in hold phase $V_{\Phi}$ goes down, and $V_{\bar{\phi}}$ goes up.

In evaluation phase, when the output logic is low and $\mathrm{P}$ network is on, $C_{L}$ is charged through pMOS (P1) and $\mathrm{P}$ network resulting in the high logic at output. When output logic is high and $\mathrm{N}$ network is on, discharging and recycling of charges to the power clock $\left(V_{\bar{\phi}}\right)$ via nMOS (N1) and N network occur, resulting in the low output logic.

In hold phase, $V_{\Phi}$ swings down, and $V_{\bar{\phi}}$ swings up, and when they reaches below the threshold voltage, both the MOSFETs P1 and N1 turn off; thus no transitions occur at the output. Due to the hold phase, dynamic switching and hence energy dissipation are reduced.

3.3. Estimation of Energy Dissipation. The energy dissipated in the proposed inverter (in Figure 2, P network is replaced by pMOS and N network by nMOS) is calculated for a triangular supply voltage (for simplicity) as shown in Figure 3 using the approximate expression.

\subsubsection{Energy Dissipation in Proposed Inverter during Charging.} When $\mathrm{P}$ network is on (pMOS), and as $V_{\Phi}$ increases to $V_{\mathrm{DD}}$ and $V_{\bar{\phi}}$ decreases to $0 \mathrm{~V}$ or vice versa, load capacitor is charged through charging transistors P1 and pMOS:

$$
\begin{aligned}
V_{\Phi} & =\frac{3 V_{\mathrm{DD}}}{4}+\frac{2 V_{\mathrm{DD}}}{T} t, & \text { when } 0 \leq t \leq \frac{T}{8} \\
& =\frac{3 V_{\mathrm{DD}}}{4}-\frac{2 V_{\mathrm{DD}}}{T} t, & \text { when } \frac{T}{8} \leq t \leq \frac{3 T}{8} \\
& =-\frac{V_{\mathrm{DD}}}{4}+\frac{2 V_{\mathrm{DD}}}{T} t, & \text { when } \frac{3 T}{8} \leq t \leq \frac{T}{2} .
\end{aligned}
$$




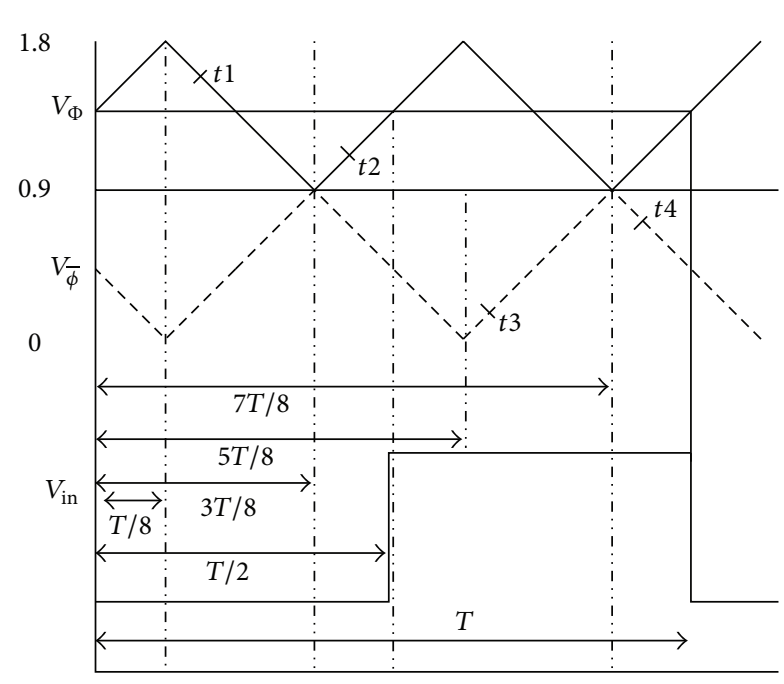

FIGURE 3: Energy dissipation estimation in IQSERL inverter.

Let us assume that the charging path resistance is $R_{1}$ and discharging path resistance is $R_{2}$. One has

$$
\begin{aligned}
& i(t)= \frac{2 V_{\mathrm{DD}}}{T} C-\frac{V_{\mathrm{DD}}}{4 T} C\left(8-3 \frac{T}{R_{1} C}\right) e^{-t / R_{1} C} \quad 0 \leq t \leq \frac{T}{8} \\
&= \frac{V_{\mathrm{DD}}}{4 T R_{1}}\left[-8 R_{1} C+\left(3 T-8 R_{1} C\right) e^{-t / R_{1} C}\right] \quad \frac{T}{8} \leq t \leq t 1 \\
&= \frac{V_{\mathrm{DD}}}{4 T R_{1}}\left[\left(4 T-8 R_{1} C\right) e^{t / R_{1} C}-8 R_{1} C\right] \quad t 1 \leq t \leq \frac{3 T}{8} \\
&= \frac{V_{\mathrm{DD}}}{4 T R_{1}}\left[8 R_{1} C+\left(T-8 R_{1} C\right) e^{t / R_{1} C}\right] \quad \frac{3 T}{8} \leq t \leq t 2 \\
&= \frac{V_{\mathrm{DD}}}{4 R_{1} T}\left[8 R_{1} C-\left(8 R_{1} C+T\right) e^{-t / R_{1} C}\right] \quad t 2 \leq t \leq \frac{T}{2}, \\
& t 1=\frac{T R_{1} C}{3 T-8 R_{1} C} \\
& t 2=\frac{3 T\left(T-4 R_{1} C\right)}{4\left(8 R_{1} C-T\right)} .
\end{aligned}
$$

Total energy dissipation in charging $E \mathrm{ch}=\int i^{2} R_{1} d t=$ $E_{1} \mathrm{ch}+E_{2} \mathrm{ch}+E_{3} \mathrm{ch}+E_{4} \mathrm{ch}+E_{5} \mathrm{ch}$,

$$
\begin{aligned}
E_{1} \mathrm{ch}= & \frac{V_{\mathrm{DD}}^{2} C^{2} R_{1}}{16 T^{2}} \\
& \times\left[\begin{array}{c}
8 T-\left(32 R_{1} C+\frac{9 T^{2}}{2 R_{1} C}-24 T\right)\left(\mathrm{e}^{-T / 4 R_{1} C}-1\right) \\
+\left(128 R_{1} C-48 T\right)\left(\mathrm{e}^{-T / 8 R_{1} C}-1\right)
\end{array}\right],
\end{aligned}
$$

$$
\begin{aligned}
& E_{2} \mathrm{ch}=\frac{V_{\mathrm{DD}}^{2}}{16 T^{2} R_{1}} \\
& \times\left[\begin{array}{c}
64 R_{1}^{2} C^{2}\left(-\frac{T}{8}+t 1\right)-\frac{R_{1} C}{2}\left(\begin{array}{c}
64 R_{1}^{2} C^{2}+9 T^{2} \\
-48 R_{1} C T
\end{array}\right) \\
\left(e^{-2 t 1 / R_{1} C}-e^{-T / 4 R_{1} C}\right)+16 R_{1}^{2} C^{2}\left(3 T-8 R_{1} C\right) \\
\left(e^{-t 1 / R_{1} C}-e^{-T / 8 R_{1} C}\right)
\end{array}\right], \\
& E_{3} \mathrm{ch}=\frac{V_{\mathrm{DD}}^{2}}{16 T^{2} R_{1}} \\
& \times\left[\begin{array}{c}
64 R_{1}^{2} C^{2}\left(\frac{3 T}{8}-t 1\right)-\frac{R_{1} C}{2}\left(\begin{array}{c}
64 R_{1}^{2} C^{2}+9 T^{2} \\
-48 R_{1} C T
\end{array}\right) \\
\left(e^{3 T / 4 R_{1} C}-e^{2 t 1 / R_{1} C}\right)-16 R_{1}^{2} C^{2}\left(3 T-8 R_{1} C\right) \\
\left(e^{3 T / 8 R_{1} C}-e^{t 1 / R_{1} C}\right)
\end{array}\right], \\
& E_{4} \mathrm{ch}=\frac{V_{\mathrm{DD}}^{2}}{16 T^{2} R_{1}} \\
& \times\left[\begin{array}{c}
64 R_{1}^{2} C^{2}\left(t 2-\frac{3 T}{8}\right)-\frac{R_{1} C}{2}\left(\begin{array}{c}
64 R_{1}^{2} C^{2}+T^{2} \\
-16 R_{1} C T
\end{array}\right) \\
\left(e^{2 t 2 / R_{1} C}-e^{3 T / 4 R_{1} C}\right)-16 R_{1}^{2} C^{2}\left(T-8 R_{1} C\right) \\
\left(e^{t 2 / R_{1} C}-e^{3 T / 8 R_{1} C}\right)
\end{array}\right], \\
& E_{5} \mathrm{ch}=\frac{V_{\mathrm{DD}}^{2}}{16 T^{2} R_{1}} \\
& \times\left[\begin{array}{c}
64 R_{1}^{2} C^{2}\left(\frac{T}{2}-t 2\right)-\frac{R_{1} C}{2}\left(\begin{array}{c}
64 R_{1}^{2} C^{2}+T^{2} \\
+16 R_{1} C T
\end{array}\right) \\
\left(e^{-T / R_{1} C}-e^{2 t 2 / R_{1} C}\right)+16 R_{1}^{2} C^{2}\left(T+8 R_{1} C\right) \\
\left(e^{-T / 2 R_{1} C}-e^{-t 2 / R_{1} C}\right)
\end{array}\right] .
\end{aligned}
$$

3.3.2. Energy Dissipation in the Inverter during Discharging. When the $\mathrm{N}$ network is on (i.e., nMOS) and P network is off, charging of the capacitor is prevented, and the load capacitor discharges through the discharging transistors N1 and nMOS. Consider

$$
\begin{aligned}
V_{\bar{\phi}} & =\frac{5 V_{\mathrm{DD}}}{4}-\frac{2 V_{\mathrm{DD}}}{T} t, & & \text { when } \frac{T}{2} \leq t \leq \frac{5 T}{8} \\
& =\frac{2 V_{\mathrm{DD}}}{T} t-\frac{5 V_{\mathrm{DD}}}{4}, & & \text { when } \frac{5 T}{8} \leq t \leq \frac{7 T}{8} \\
& =\frac{9 V_{\mathrm{DD}}}{4}-\frac{2 V_{\mathrm{DD}}}{T} t, & & \text { when } \frac{7 T}{8} \leq t \leq T,
\end{aligned}
$$

$$
\begin{array}{r}
i(t)=\frac{V_{\mathrm{DD}}}{4 R_{2} T}\left[-8 R_{2} C+\left(8 R_{2} C-5 T\right)\right] e^{t / R_{2} C} \\
\frac{T}{2} \leq t \leq \frac{5 T}{8} \\
=\frac{V_{\mathrm{DD}}}{4 R_{2} T}\left[8 R_{2} C+\left(5 T-8 R_{2} C\right)\right] e^{t / R_{2} C} \\
\frac{5 T}{8} \leq t \leq t 3
\end{array}
$$




$$
\begin{gathered}
=\frac{V_{\mathrm{DD}}}{4 R_{2} T}\left[8 R_{2} C-\left(5 T+8 R_{2} C\right)\right] e^{-t / R_{2} C} \\
t 3 \leq t \leq \frac{7 T}{8} \\
=\frac{V_{\mathrm{DD}}}{4 R_{2} T}\left[-8 R_{2} C+\left(9 T+8 R_{2} C\right)\right] e^{-t / R_{2} C} \\
\frac{7 T}{8} \leq t \leq t 4 \\
=\frac{V_{\mathrm{DD}}}{4 R_{2} T}\left[-8 R_{2} C+\left(8 R_{2} C-9 T\right)\right] e^{t / R_{2} C} \\
t 4 \leq t \leq T, \\
t 3=\frac{-25 T^{2} R_{2} C+2.5 T^{3}-8 R_{2}^{2} C^{2} T}{\left(8 R_{2} C-T\right)\left(8 R_{2} C+5 T\right)}, \\
t 4=\frac{\left(10 T-16 R_{2} C\right) t 3+7 T^{2}+36 R_{2} C T}{2\left(9 T-8 R_{2} C\right)} .
\end{gathered}
$$

Total energy dissipation in discharging, Edisch = $\int i^{2} R_{2} d t=E_{1}$ disch $+E_{2}$ disch $+E_{3}$ disch $+E_{4}$ disch $+E_{5}$ disch

$$
\begin{aligned}
& E_{1} \text { disch }=\frac{V_{\mathrm{DD}}^{2}}{16 T^{2} R_{2}} \\
& \times\left[\begin{array}{c}
64 R_{2}^{2} C^{2}\left(\frac{5 T}{8}-\frac{T}{2}\right)+\frac{R_{2} C}{2}\left(\begin{array}{c}
64 R_{2}^{2} C^{2}+25 T^{2} \\
-80 R_{2} C T
\end{array}\right) \\
\left(e^{5 T / 4 R_{2} C}-e^{T / R_{2} C}\right)-16 R_{2}^{2} C^{2}\left(8 R_{2} C-5 T\right) \\
\left(e^{5 T / R_{2} C}-e^{T / 2 R_{2} C}\right)
\end{array}\right], \\
& E_{2} \text { disch }=\frac{V_{\mathrm{DD}}^{2}}{16 T^{2} R_{2}} \\
& \times\left[\begin{array}{c}
64 R_{2}^{2} C^{2}\left(t 3-\frac{5 T}{8}\right)+\frac{R_{2} C}{2}\left(\begin{array}{c}
64 R_{2}^{2} C^{2}+25 T^{2} \\
-80 R_{2} C T
\end{array}\right) \\
\left(e^{2 t 3 / R_{2} C}-e^{5 T / 4 R_{2} C}\right)+16 R_{2}^{2} C^{2}\left(5 T-8 R_{2} C\right) \\
\left(e^{t 3 / R_{2} C}-e^{5 T / 8 R_{2} C}\right)
\end{array}\right], \\
& E_{3} \operatorname{disch}=\frac{V_{\mathrm{DD}}^{2}}{16 T^{2} R_{2}} \\
& \times\left[\begin{array}{c}
64 R_{2}^{2} C^{2}\left(\frac{7 T}{8}-t 3\right)+\frac{R_{2} C}{2}\left(\begin{array}{c}
64 R_{2}^{2} C^{2}+25 T^{2} \\
-80 R_{2} C T
\end{array}\right) \\
\left(e^{-7 T / 4 R_{2} C}-e^{-2 t 3 / R_{2} C}\right)+16 R_{2}^{2} C^{2}\left(8 R_{2} C+5 T\right) \\
\left(e^{-7 T / 8 R_{2} C}-e^{-t 3 / R_{2} C}\right)
\end{array}\right], \\
& E_{4} \text { disch }=\frac{V_{\mathrm{DD}}^{2}}{16 T^{2} R_{2}} \\
& \times\left[\begin{array}{c}
64 R_{2}^{2} C^{2}\left(t 4-\frac{7 T}{8}\right)+\frac{R_{2} C}{2}\left(\begin{array}{c}
64 R_{2}^{2} C^{2}+81 T^{2} \\
+144 R_{2} C T
\end{array}\right) \\
\left(e^{-2 t 4 / R_{2} C}-e^{-7 T / 4 R_{2} C}\right)-16 R_{2}^{2} C^{2}\left(8 R_{2} C+9 T\right) \\
\left(e^{-t 4 / R_{2} C}-e^{-7 T / 8 R_{2} C}\right)
\end{array}\right],
\end{aligned}
$$

$$
\begin{aligned}
E_{5} \text { disch }= & \frac{V_{\mathrm{DD}}^{2}}{16 T^{2} R_{2}} \\
& \times\left[\begin{array}{c}
64 R_{2}^{2} C^{2}(T-t 4)+\frac{R_{2} C}{2}\left(\begin{array}{c}
64 R_{2}^{2} C^{2}+81 T^{2} \\
-144 R_{2} C T
\end{array}\right) \\
\left(e^{2 T / R_{2} C}-e^{2 t 4 / R_{2} C}\right)-16 R_{2}^{2} C^{2}\left(8 R_{2} C-9 T\right) \\
\left(e^{T / R_{2} C}-e^{t 4 / R_{2} C}\right)
\end{array}\right] .
\end{aligned}
$$

\section{Results and Analysis}

\subsection{Proposed IQSERL Inverter Circuit}

4.1.1. Driving Ability. Figure 4 shows the driving ability of the proposed logic in eight-stage inverter chain. We tested the delay at each stage of the inverter chain (with simulation parameters as W/L of pMOS 540/180 nm and nMOS 240/ $180 \mathrm{~nm}$ and $50 \mathrm{MHz}$ input rate and $200 \mathrm{MHz}$ power clock rate) one by one and observed that delay is significantly reduced in comparison to the QSERL circuits as was observed in Figure 1(b). It may be observed that due to the very small amount of delay at each stage, correct output logics are observed throughout the eight-stages. Our attempt for delay reduction over QSERL is to ensure that the supply voltage is split-level sinusoidal instead of sinusoidal power clock, since it was observed that split-level power clock based circuits have lesser delay than the others [15]. Further by sizing of charging pMOS transistors (width is increased, i.e., $540 \mathrm{~nm}$ ) and by increasing the frequency ratio (power clock frequency to the input frequency 4 times), delay is reduced.

4.1.2. Power Efficiency with Frequency. The input and supply frequencies varied simultaneously (in IQSERL supply frequency is four times the input frequency for better performance) from $0.01 \mathrm{MHz}$ to $50 \mathrm{MHz}$, and load capacitance at each stage is set to $10 \mathrm{fF}$ as shown in Figure 5. As frequency increases, power dissipation of all three types of circuits increases, whereas proposed inverter has lesser power dissipation at each frequency in comparison to CMOS and QSERL. However, after $33.3 \mathrm{MHz}$ output levels of QSERL become incorrect and cause lesser power dissipation than the proposed IQSERL. We may also note that QSERL has larger power dissipation than CMOS at some points which again prove the poor performance of QSRL. Also a continuous decrease in percentage power saving of IQSERL inverter over CMOS and QSERL with frequency is observed.

From the Table 1 we observe that the proposed inverter has lowest power dissipation, lower delay than QSERL, and comparable delay to the CMOS at all the observed frequencies.

4.1.3. Power Efficiency with Load Capacitance. We tested the inverters by adding extra capacitive load at the output node one by one from $10 \mathrm{fF}$ to $300 \mathrm{fF}$ as shown in Figure 6. Clock and data rate are kept fixed at $4 \mathrm{MHz}$ and $1 \mathrm{MHz}$, respectively. When load capacitance is increased, power dissipation of all three types of circuits increases correspondingly; however, 


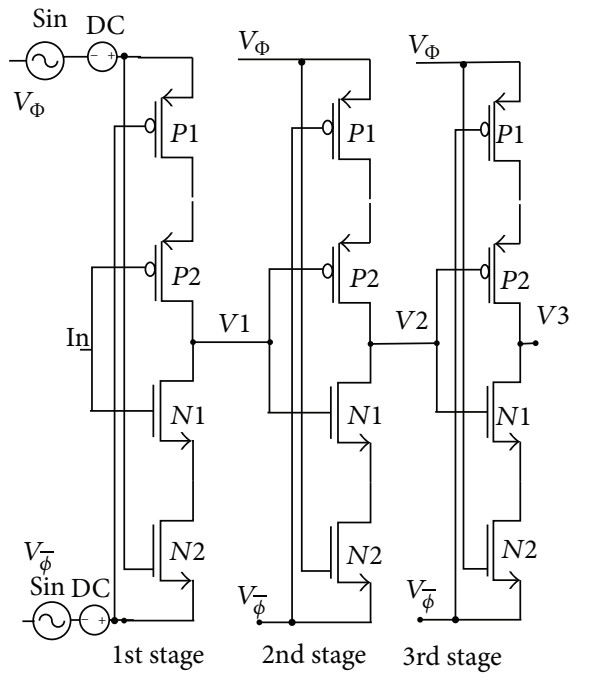

(a)

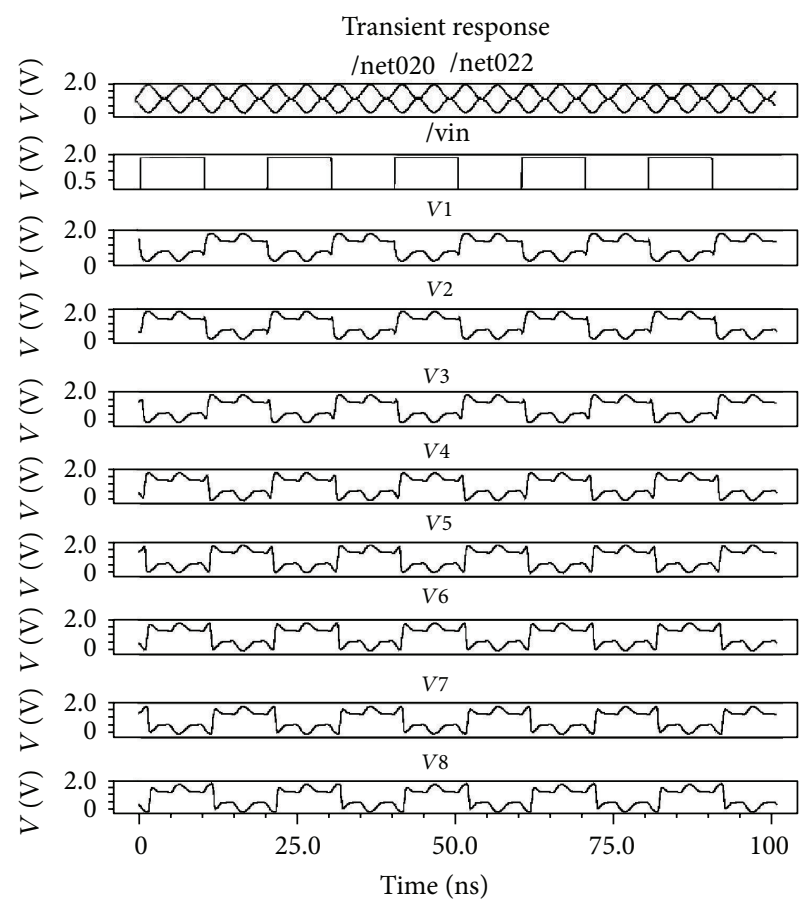

(b)

FIGURE 4: (a) IQSERL eight-stage inverter chain, (b) transient response.

TABLE 1: Comparison of power, delay, and PDP of 8-inverter chain in 10 cycles of charging and discharging.

\begin{tabular}{|c|c|c|c|c|c|c|c|}
\hline \multicolumn{8}{|c|}{ Power dissipation $(\mu \mathrm{W})$} \\
\hline Frequency $(\mathrm{MHz})$ & 0.01 & 0.1 & 1 & 10 & 20 & 33.3 & 50 \\
\hline CMOS & 0.09 & 0.12 & 0.40 & 3.32 & 6.6 & 10.9 & 16.4 \\
\hline QSERL & 0.02 & 0.10 & 0.66 & 3.69 & 6.2 & 8.95 & 11.7 \\
\hline IQSERL & 0.01 & 0.02 & 0.13 & 1.92 & 4.8 & 8.92 & 15.2 \\
\hline \multicolumn{8}{|c|}{ Delay (ns) } \\
\hline CMOS & 55.4 & 3.15 & 1.37 & 1.52 & 1.5 & 1.48 & 1.47 \\
\hline QSERL & 4210 & 522 & 64.4 & 9.38 & 5.69 & 4.10 & 3.29 \\
\hline IQSERL & 1.92 & 6.24 & 4.53 & 2.56 & 2.07 & 1.8 & 1.68 \\
\hline \multicolumn{8}{|c|}{ PDP (fJ) } \\
\hline CMOS & 4.99 & 0.38 & 0.55 & 5.05 & 9.9 & 16.1 & 24.1 \\
\hline QSERL & 84.2 & 52.2 & 42.5 & 34.6 & 35.3 & 36.7 & 38.5 \\
\hline IQSERL & 0.02 & 0.12 & 0.59 & 4.92 & 9.94 & 16.0 & 25.5 \\
\hline
\end{tabular}

our proposed inverter has good power efficiency than CMOS and QSERL at each point. When load capacitance increases, percentage power saving decreases and power saving of IQSERL to the QSERL is more than the power saving to the CMOS in lower frequency range.

Table 2 shows that the proposed IQSERL inverter has lowest power dissipation, lower delay than QSERL, and comparable delay to the CMOS at all the observed load capacitances.

4.1.4. Layout of Proposed IQSERL Inverter. The layout of proposed inverter has been drawn as shown in Figure 7. The chip area of IQSERL inverter has been characterized $\left(20.7 \mu \mathrm{m}^{2}\right)$ to be higher than the CMOS $\left(14.54 \mu \mathrm{m}^{2}\right)$ due to the extra nMOS

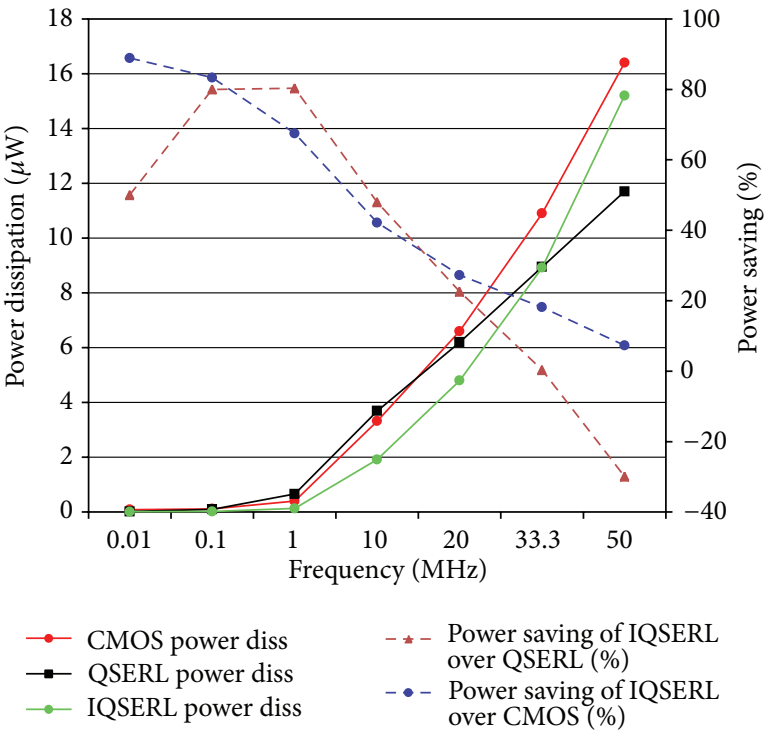

FIGURE 5: Simulation results of the performance comparison of 8inverter chain with frequency.

and pMOS in discharging/charging paths. Thus the proposed logic requiring fewer transistors is comparable to the CMOS.

4.2. Proposed IQSERL Multiplier Circuit. In this section a $4 \times 4$ bit IQSERL array multiplier is designed and simulated to show the usefulness of the new logic family for large circuits. It consists of 16 IQSERL AND gates, 4 IQSERL half adders, 8 IQSERL full adders, and 8 IQSERL D flip flops as shown 
TABLE 2: Comparison of power, delay, and PDP of 8-inverter chain with load capacitance at $f_{i}=1 \mathrm{MHz}$.

\begin{tabular}{|c|c|c|c|c|c|c|c|}
\hline \multicolumn{8}{|c|}{ Power dissipation $(\mu \mathrm{W})$} \\
\hline Capacitance (fF) & 20 & 30 & 50 & 100 & 200 & 250 & 300 \\
\hline CMOS & 0.66 & 0.93 & 1.4 & 2.7 & 5.4 & 6.8 & 8.1 \\
\hline QSERL & 0.99 & 1.3 & 1.8 & 3.1 & 5.31 & 6.31 & 7.2 \\
\hline IQSERL & 0.23 & 0.35 & 0.61 & 1.3 & 3.34 & 4.4 & 5.7 \\
\hline \multicolumn{8}{|c|}{ Delay (ns) } \\
\hline CMOS & 2.7 & 4.1 & 6.6 & 12.8 & 24.9 & 30.9 & 36.9 \\
\hline QSERL & 69.4 & 73.1 & 79.2 & 91 & 109 & 117 & 124 \\
\hline IQSERL & 7.18 & 9.5 & 13.5 & 21.8 & 35 & 40.8 & 46.3 \\
\hline \multicolumn{8}{|c|}{ PDP (fJ) } \\
\hline CMOS & 1.78 & 3.8 & 9.24 & 34.5 & 134 & 210 & 298 \\
\hline QSERL & 68.7 & 95 & 142 & 282 & 578 & 738 & 892 \\
\hline IQSERL & 1.65 & 3.32 & 8.24 & 28.3 & 116 & 179 & 263 \\
\hline
\end{tabular}
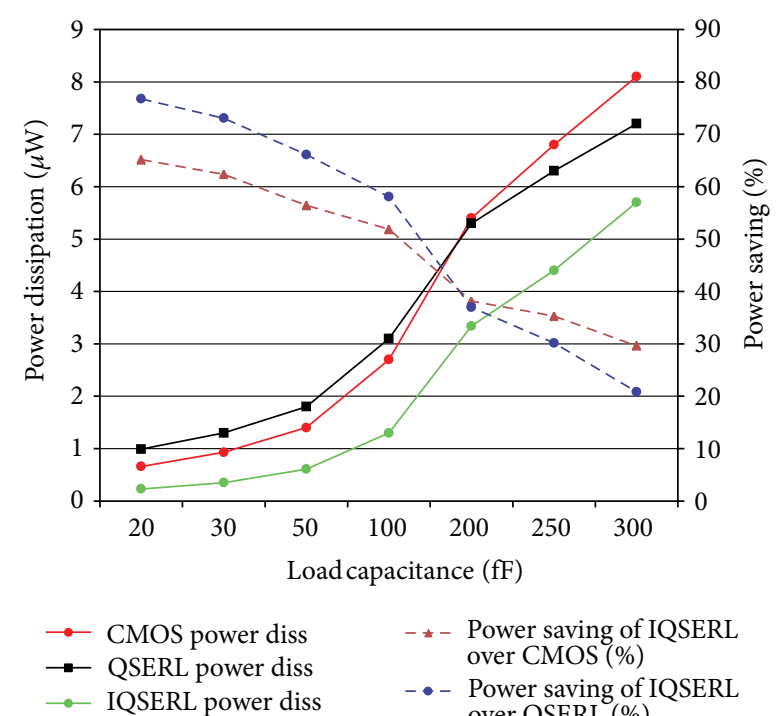

$$
\begin{aligned}
-\star- & \text { Power saving of IQSERL } \\
& \text { over CMOS (\%) } \\
-\bullet- & \text { Power saving of IQSERL } \\
& \text { over QSERL (\%) }
\end{aligned}
$$

FIGURE 6: Simulation results of the performance comparison of 8inverter chain with load capacitance.

in Figure 8(a). D flip flops are used to store the 8-bit signals. The simulation is done with simulation parameters as $W / L$ of pMOS 540/180 nm and nMOS $240 / 180 \mathrm{~nm}$, and input rate was kept four times the power clock rate (in IQSERL). From Figure 8(b), the simulated input and output timing waveforms verify the 4-bit multiplier logic.

Simulation results for the proposed IQSERL multiplier are compared with CMOS and recently reported (QSERL) multipliers [17-19] using the same logic implementation, equal switching probability of inputs, and the same simulation condition. The input and supply frequencies are varied simultaneously (keeping the supply frequency four times the input frequency in IQSERL) from $0.1 \mathrm{MHz}$ to $20 \mathrm{MHz}$, and corresponding power dissipations for the multipliers have been measured. IQSERL multiplier has significant power saving to the CMOS and QSERL multipliers. As frequency increases, the power saving of IQSERL to the CMOS and
TABLE 3: Comparison of transistor counts of $4 \times 4$ bit array multiplier.

\begin{tabular}{lc}
\hline Design style & Number of transistors \\
\hline CMOS & 744 \\
QSERL & 1432 \\
IQSERL & 1056 \\
\hline
\end{tabular}

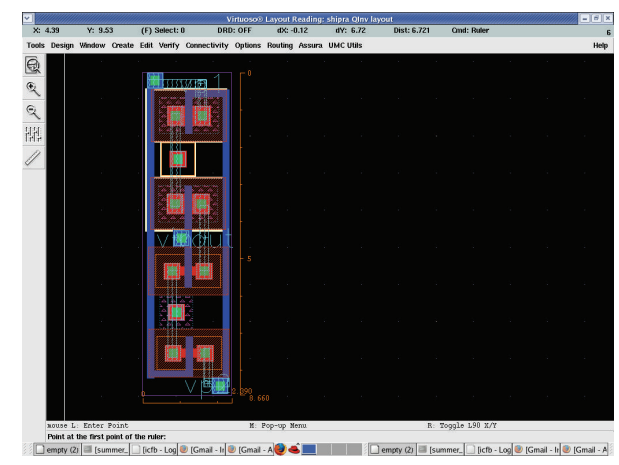

FIGURE 7: Layout of proposed IQSERL inverter circuit.

QSERL multipliers decreases as shown in Figure 9. At $0.05 \mathrm{MHz} 98 \%$ saving in power as compared to the QSERL multiplier and $94 \%$ power saving to the CMOS multiplier are obtained. At $20 \mathrm{MHz}$ the power saving to the QSERL multiplier is $7 \%$ whereas to the CMOS multiplier is $11 \%$. As frequency goes higher, the resistive dissipation (which is proportional to $R C / T$ ) increases, so power saving is less.

After a certain high frequency (here $20 \mathrm{MHz}$ ), incorrect output logics are obtained, so for larger complex circuits, our IQSERL circuit is limited in some high frequency range. Also from the layout of proposed IQSERL inverter circuit as in Figure 7, we can generalize it for multiplier circuit also that, for a given performance, devices can be smaller for a CMOS multiplier circuit than the IQSERL multiplier circuit. Hence the proposed IQSERL circuit is more suitable for some specific applications where speed and area are not critical. Since a $4 \times 4$ bit array multiplier is a large complex circuit, so number of transistors on a chip (transistor count) is also an important parameter to be discussed. In Table 3, we have given the comparative study of transistor count of our proposed multiplier with others.

It may be observed that the proposed IQSERL logic requiring fewer transistors is comparable to the CMOS, whereas IQSERL logic have less transistor counts than QSERL. Thus it needs less complex layout design and can be used to build larger circuits on a single chip.

\section{Conclusion}

The simulation results and comparative performance evaluation revealed that power dissipation in the IQSERL logic family is considerably lower than the CMOS and the reported adiabatic (QSERL) family; thus the proposed IQSERL family outperforms and provides almost $20 \%$ or greater power saving over CMOS and QSERL up to $50 \mathrm{MHz}$ for the IQSERL 


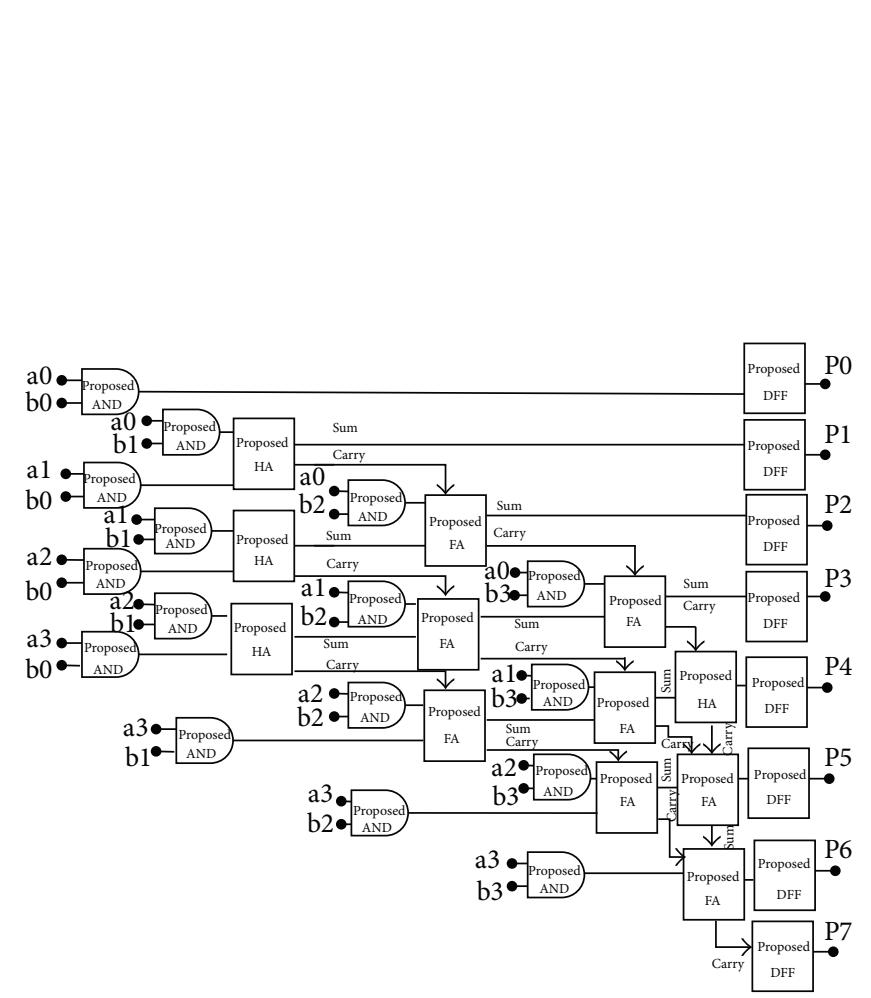

(a)

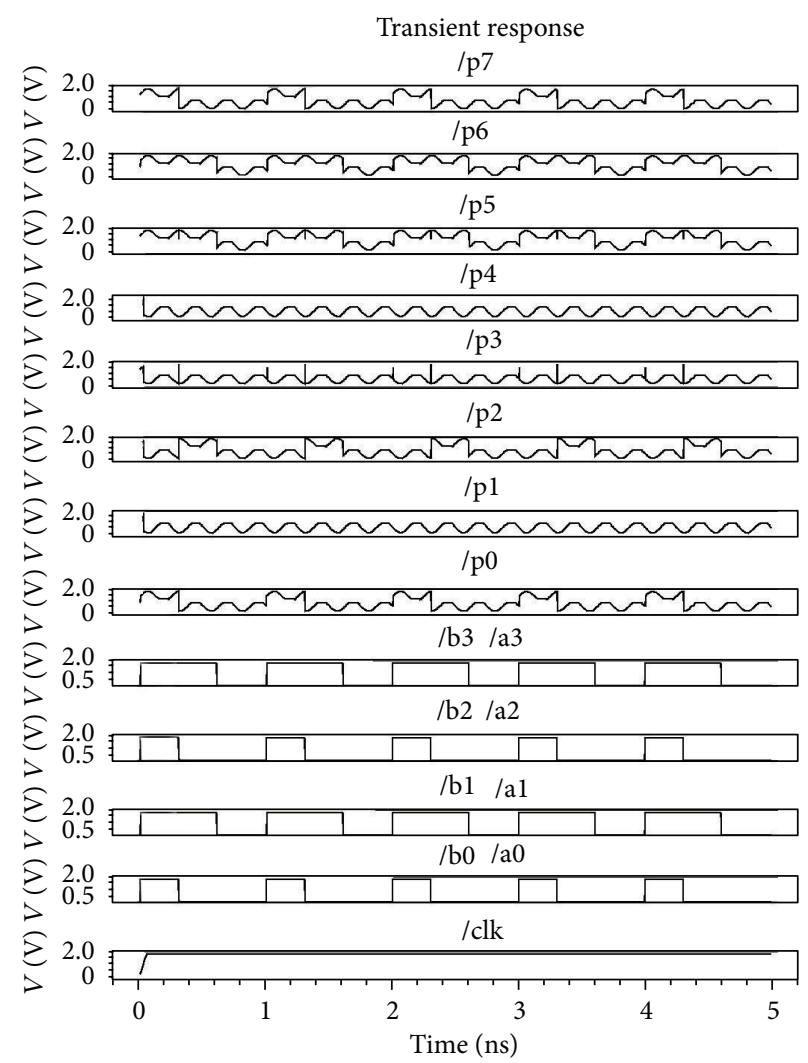

(b)

FIGURE 8: (a) IQSERL $4 \times 4$ bit array multiplier circuit, (b) transient response.

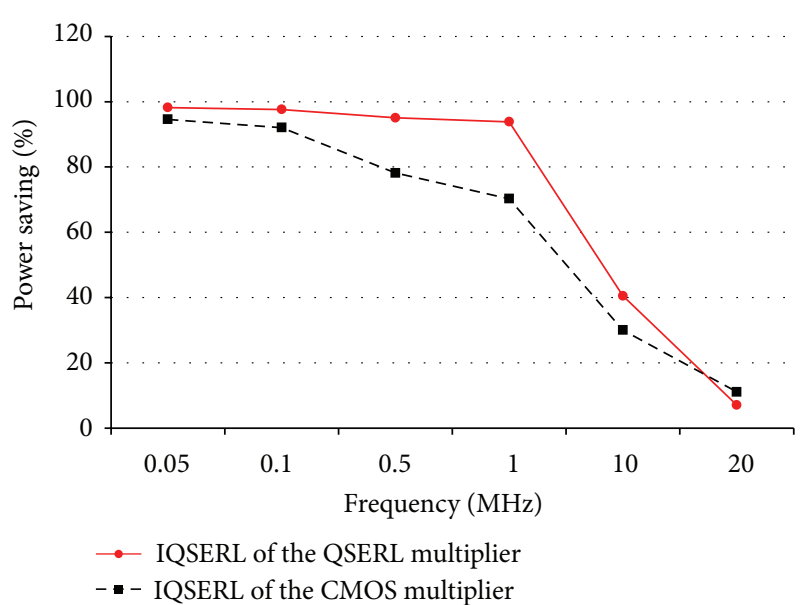

FIGURE 9: Simulation results of the performance comparison of $4 \times 4$ bit array multipliers with frequency.

inverter. A $4 \times 4$ bit array IQSERL multiplier circuits has almost $11 \%$ or greater power saving to the CMOS and $7 \%$ or greater power saving to the QSERL multiplier up to $20 \mathrm{MHz}$. The layout of IQSERL inverter circuit (area $20.7 \mu \mathrm{m}^{2}$ ) is larger than CMOS inverter (area $14.54 \mu \mathrm{m}^{2}$ ) which reveals that devices can be smaller for a CMOS circuit than the IQSERL circuit. Thus the proposed IQSERL circuit is more suitable for some specific applications where speed and area are not critical.

\section{Acknowledgments}

The authors duly acknowledged with gratitude the support from Ministry of Communications and Information Technology, DIT, Government of India, New Delhi, through Special Manpower Development Program in VLSI and related Software's Phase-II (SMDP-II) Project in E and CE Department, MNNIT Allahabad, India.

\section{References}

[1] S. Wairya, R. K. Nagaria, and S. Tiwari, "Performance analysis of high speed hybrid CMOS full adder circuits for low voltage VLSI design," VLSI Design, Article ID 173079, pp. 1-18, 2012.

[2] V. K. De and J. D. Meindl, "Opportunities for non-dissipative computation," in Proceedings of the 9th Annual IEEE International ASIC Conference and Exhibit, pp. 297-300, September 1996.

[3] W. C. Athas, L. Svensson, J. G. Koller, N. Tzartzanis, and E. Y.-C. Chou, "Low-power digital systems based on adiabatic-switching principles," IEEE Transactions on Very Large Scale Integration (VLSI) Systems, vol. 2, no. 4, pp. 398-407, 1994.

[4] A. G. Dickinson and J. S. Denker, "Adiabatic dynamic logic," IEEE Journal of Solid-State Circuits, vol. 30, no. 3, pp. 311-315, 1995.

[5] V. S. Sathe, J.-Y. Chueh, and M. C. Papaefthymiou, "Energyefficient GHz-class charge-recovery logic," IEEE Journal of Solid-State Circuits, vol. 42, no. 1, pp. 38-46, 2007. 
[6] S. Wisetphanichkij and K. Dejhan, "The combinational and sequential adiabatic circuit design and its applications," Circuits, Systems, and Signal Processing, vol. 28, no. 4, pp. 523-534, 2009.

[7] M.-E. Hwang, A. Raychowdhury, and K. Roy, "Energy-recovery techniques to reduce on-chip power density in molecular nanotechnologies," IEEE Transactions on Circuits and Systems I, vol. 52, no. 8, pp. 1580-1589, 2005.

[8] P. Wang and J. Yu, "Design of two-phase sinusoidal power clock and clocked transmission gate adiabatic logic circuit," Journal of Electronics, vol. 24, no. 2, pp. 225-231, 2007.

[9] Y. Ye and K. Roy, "QSERL: quasi-static energy recovery logic," IEEE Journal of Solid-State Circuits, vol. 36, no. 2, pp. 239-248, 2001.

[10] N. S. S. Reddy, M. Satyam, and K. L. Kishore, "Cascadable adiabatic logic circuits for low-power applications," IET Circuits, Devices and Systems, vol. 2, no. 6, pp. 518-526, 2008.

[11] Y. Moon and D.-K. Jeong, "An efficient charge recovery logic circuit," IEEE Journal of Solid-State Circuits, vol. 31, no. 4, pp. 514-522, 1996.

[12] D. Maksimović, V. G. Oklobdžija, B. Nikolić, and K. W. Current, "Clocked CMOS adiabatic logic with integrated single-phase power-clock supply," IEEE Transactions on Very Large Scale Integration (VLSI) Systems, vol. 8, no. 4, pp. 460-463, 2000.

[13] A. Blotti and R. Saletti, "Ultralow-power adiabatic circuit semicustom design," IEEE Transactions on Very Large Scale Integration (VLSI) Systems, vol. 12, no. 11, pp. 1248-1253, 2004.

[14] C.-S. A. Gong, M.-T. Shiue, C.-T. Hong, and K.-W. Yao, "Analysis and design of an efficient irreversible energy recovery logic in $0.18 \mu \mathrm{m}$ CMOS," IEEE Transactions on Circuits and Systems, vol. 55, no. 9, pp. 2595-2607, 2008.

[15] N. Anuar, Y. Takahashi, and T. Sekine, "Two phase clocked adiabatic static CMOS logic and its logic family," Journal of Semiconductor Technology and Science, vol. 10, no. 1, pp. 1-10, 2010.

[16] S. Upadhyay, R. K. Nagaria, and R. A. Mishra, "Complementary energy path adiabatic logic based full adder circuit," A Journal of World Academy of Science Engineering \& Technology, vol. 66, pp. 161-167, 2012.

[17] M. Khatir, A. Ejlali, and A. Moradi, "Improving the energy efficiency of reversible logic circuits by the combined use of adiabatic styles," Integration, the VLSI Journal, vol. 44, pp. 12-21, 2011.

[18] N. A. Nayan, Y. Takahashi, and T. Sekine, "LSI implementation of a low-power $4 \times 4$-bit array two-phase clocked adiabatic static CMOS logic multiplier," Microelectronics Journal, vol. 43, no. 4, pp. 244-249, 2012.

[19] S. Upadhyay, R. A. Mishra, R. K. Nagaria, and S. P. Singh, "DFAL: diode free adiabatic logic circuits," International Scholarly Research Network, vol. 2013, Article ID 673601, 12 pages, 2013. 

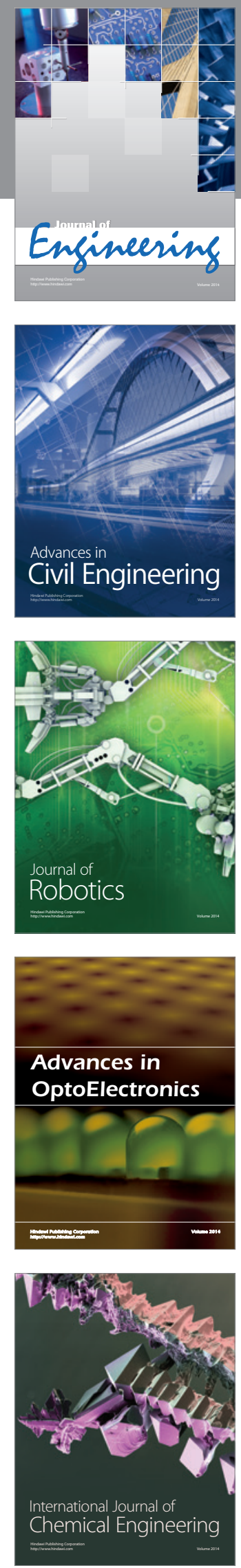

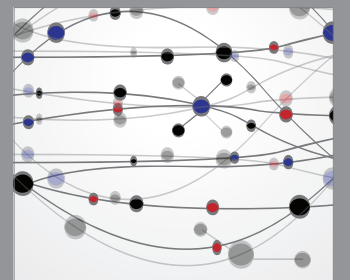

The Scientific World Journal
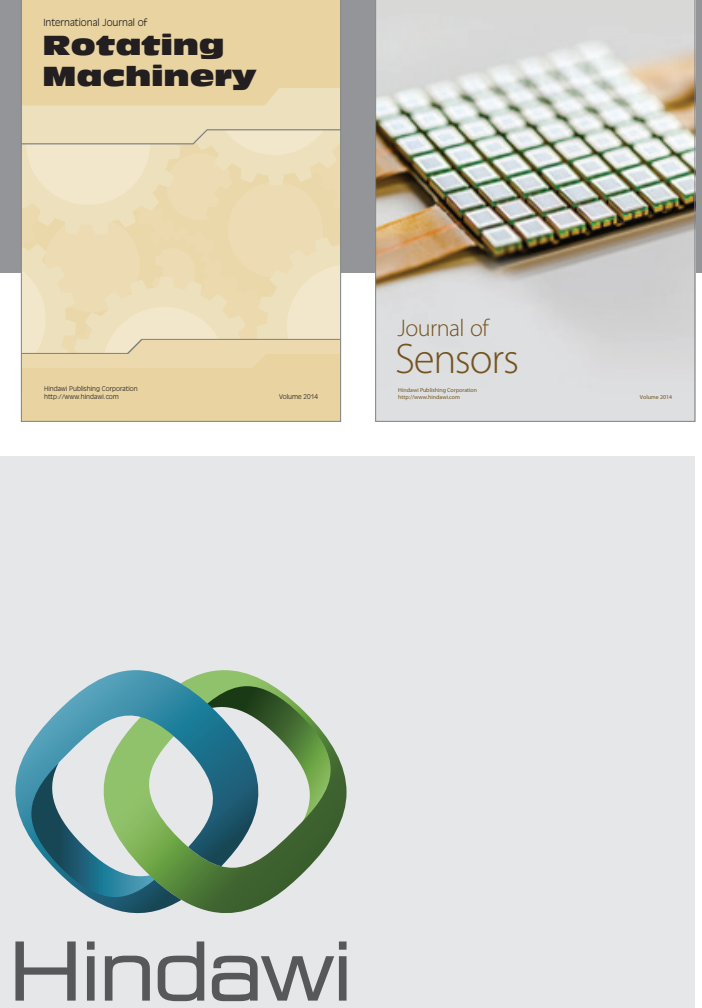

Submit your manuscripts at http://www.hindawi.com
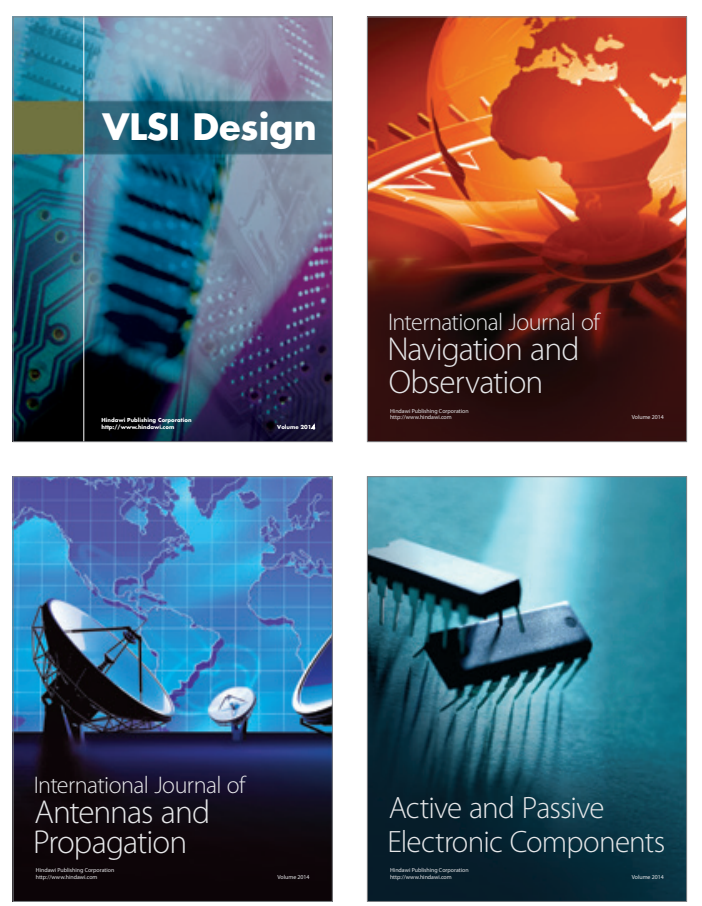
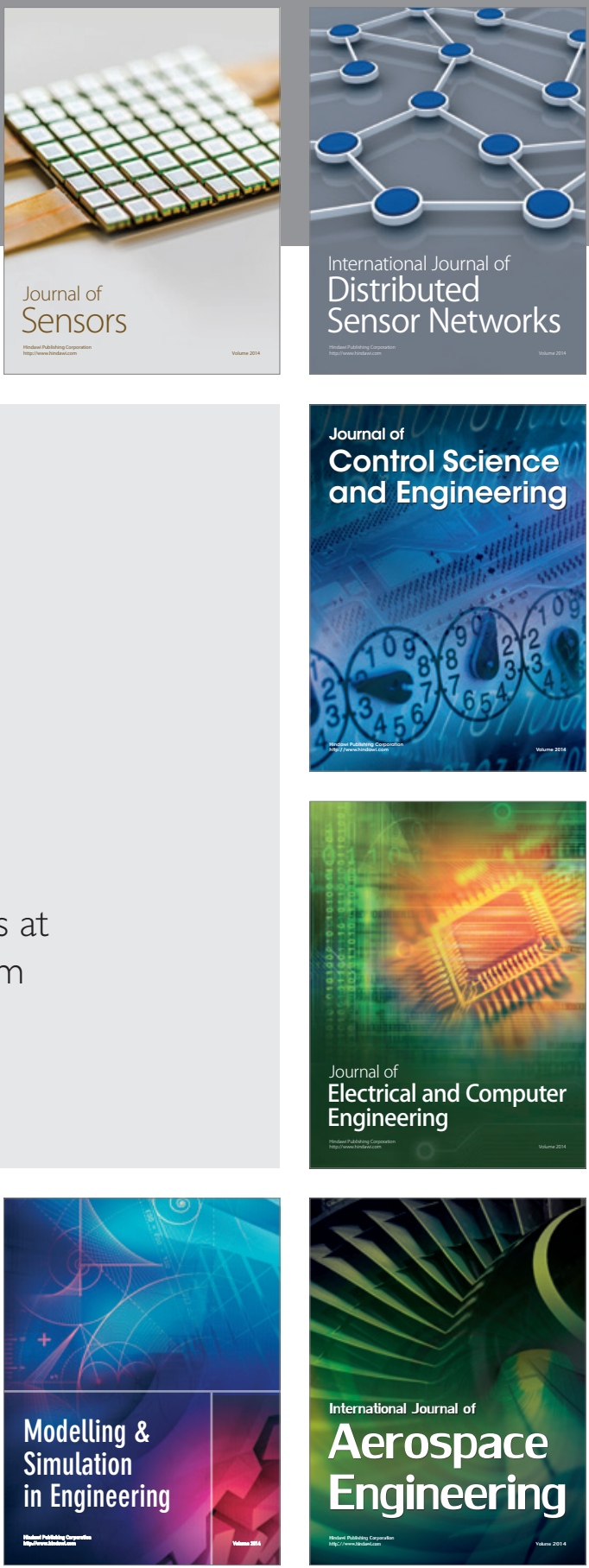

Journal of

Control Science

and Engineering
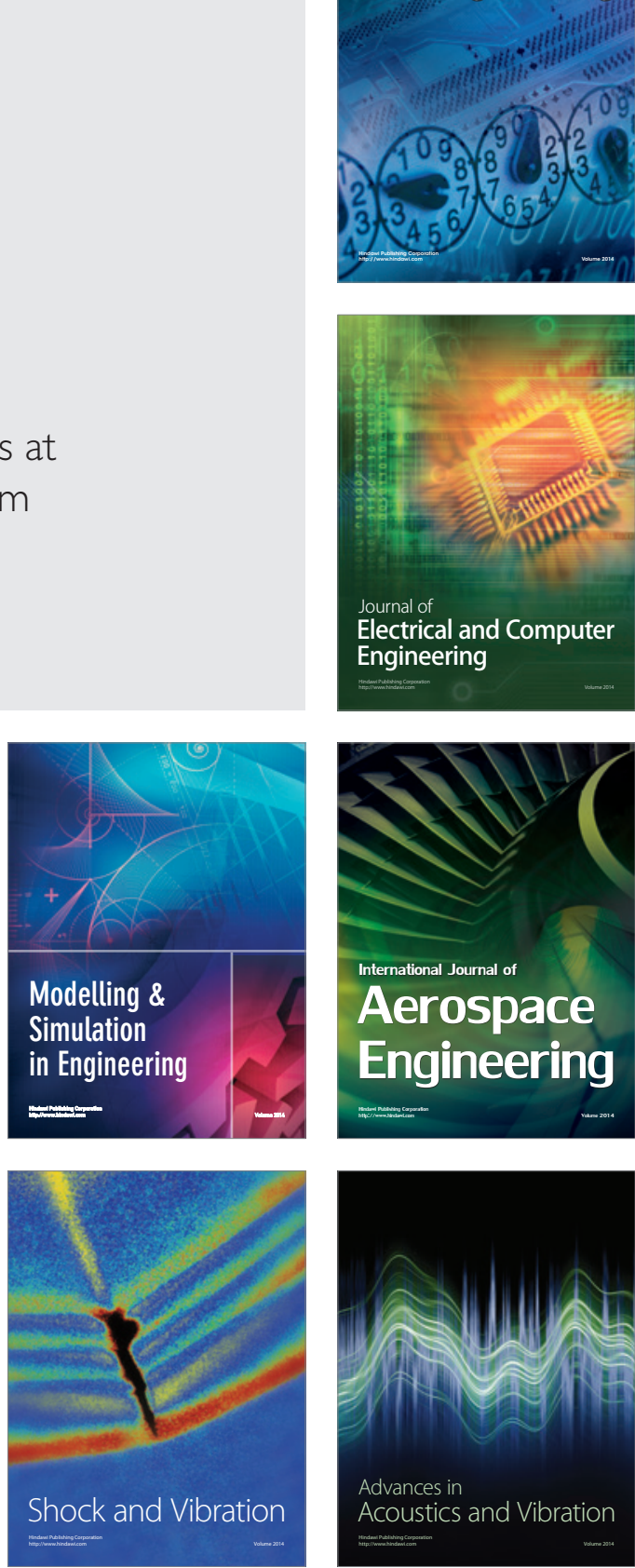